University of Wollongong

Research Online

Faculty of Engineering and Information

Faculty of Engineering and Information

Sciences - Papers: Part A

Sciences

$1-1-2015$

\title{
Bridge deterioration modeling by Markov Chain Monte Carlo (MCMC) simulation method
}

\author{
N.K. Walgama Wellalage \\ University of Wollongong,wwnk807@uowmail.edu.au \\ Tieling Zhang \\ University of Wollongong, tieling@uow.edu.au \\ Richard Dwight \\ University of Wollongong, radwight@uow.edu.au \\ Khaled El-Akruti \\ University of Wollongong, khaled@uow.edu.au
}

Follow this and additional works at: https://ro.uow.edu.au/eispapers

Part of the Engineering Commons, and the Science and Technology Studies Commons

\footnotetext{
Research Online is the open access institutional repository for the University of Wollongong. For further information
} contact the UOW Library: research-pubs@uow.edu.au 


\title{
Bridge deterioration modeling by Markov Chain Monte Carlo (MCMC) simulation method
}

\author{
Abstract \\ There are over 10 thousands rail bridges in Australia that were made of different materials and \\ constructed at different years. Managing thousands of bridges has become a real challenge for rail bridge \\ engineers without having a systematic approach for decision making. Developing best suitable \\ deterioration models is essential in order to implement a comprehensive Bridge Management System \\ (BMS). In State Based Markov Deterioration (SBMD) modeling, the main task is to estimate Transition \\ Probability Matrixes (TPMs). In this study, Markov Chain Monte Carlo (MCMC) simulation method is \\ utilized to estimate TPMs of railway bridge elements by overcoming some limitations of conventional \& \\ nonlinear optimization-based TPM estimation methods. The bridge inventory data over 15 years of 1000 \\ Australian railway bridges were reviewed \& contribution factors for railway bridge deterioration were \\ identified. MCMC simulation models were applied at bridge network level. Results show that TPMs \\ corresponding to critical bridge elements can be obtained by Metropolis-Hasting Algorithm (MHA) coded \\ in MATLAB program until it converges to stationary transition probability distributions. The predicted \\ condition state distributions of selected bridge element group were tested by statistical hypothesis tests \\ to validate the suitability of bridge deterioration models developed.

\section{Disciplines} \\ Engineering | Science and Technology Studies

\section{Publication Details} \\ Wellalage, N. Walgama, Zhang, T., Dwight, R. \& El-Akruti, K. (2015). Bridge deterioration modeling by \\ Markov Chain Monte Carlo (MCMC) simulation method. In P. W. Tse, J. Mathew, K. Wong, R. Lam \& C. N. \\ Ko (Eds.), Proceedings of the 8th World Congress on Engineering Asset Management (WCEAM 2013) \& \\ the 3rd International Conference on Utility Management \& Safety (pp. 545-556). Switzerland: Springer \\ International Publishing.
}




\title{
Bridge Deterioration Modeling by Markov Chain Mon- te Carlo (MCMC) Simulation Method
}

\author{
W.W.N. Karunarathna ${ }^{1}$, Tieling Zhang ${ }^{2}$, Richard Dwight ${ }^{3}$ and Khaled El- \\ Akruti $^{4}$
}

\begin{abstract}
There are over 10 thousands rail bridges in Australia that were made of different materials and constructed at different years. Managing thousands of bridges has become a real challenge for rail bridge engineers without having a systematic approach for decision making. Developing best suitable deterioration models is essential in order to implement a comprehensive Bridge Management System (BMS). In State Based Markov Deterioration (SBMD) modeling, the main task is to estimate Transition Probability Matrixes (TPMs). In this study, Markov Chain Monte Carlo (MCMC) simulation method is utilized to estimate TPMs of railway bridge elements by overcoming some limitations of conventional \& nonlinear optimization-based TPM estimation methods. The bridge inventory data over 15 years of 1000 Australian railway bridges were reviewed \& contribution factors for railway bridge deterioration were identified. MCMC simulation models were applied at bridge network level. Results show that TPMs corresponding to critical bridge elements can be obtained by Metropolis-Hasting Algorithm (MHA) coded in MATLAB program until it converges to stationary transition probability distributions. The predicted condition state distributions of selected bridge element group were tested by statistical hypothesis tests to validate the suitability of bridge deterioration models developed.
\end{abstract}

\footnotetext{
${ }^{1}$ W.W.N. Karunarathna

University of Wollongong, Wollongong, NSW 2522, Australia

e-mail:wwnk807@uowmail.edu.au

${ }^{2}$ T. Zhang $(\bowtie)$

University of Wollongong, Wollongong, NSW 2522, Australia

e-mail: tieling@uow.edu.au

${ }^{3}$ R. Dwight

University of Wollongong, Wollongong, NSW 2522, Australia

e-mail: radwight@uow.edu.au

${ }^{4} \mathrm{~K}$. El-Akruti

University of Wollongong, Wollongong, NSW 2522, Australia

e-mail:khaled@uow.edu.au
} 


\subsection{Introduction and Background}

Bridge inspection data consist of condition ratings of main components such as superstructure, deck, substructure, etc., or sub key components. Although the deterioration processes of bridge components are continuous, discrete condition rating are used to measure the level of deterioration of components to reduce the complexity of the continuous condition monitoring [1]. Ratings are usually assigned on different scales by different organizations and, inspections are normally conducted once in every year or two years. For an example, Federal Highway Administration (FHWA) in USA uses range from 0 to 9 whereas railway bridge organizations in Australia assigns ratings on a scale of 1 to 6 or 1 to 4 . Inspections are normally conducted once in every year or two years if reliable bridge condition rating data are available for relatively long period of time that can be used to develop bridge component deterioration models [1], [2] \& [3]. Bridge deterioration models are used to predict the future condition states of bridge components/bridges and those are essential components of any promising Bridge Management System (BMS).

There are approximately 15,000 bridges in Australia's rail network. These bridges are made of different materials, constructed at different years. Further-

more, they are subjected to different magnitudes, frequencies and distribution of rail loading and exposed to different environmental categories; inspected and maintained by separate organizations with various inspection and maintenance standards. These uncertainties emphasize the need of probabilistic deterioration models over deterministic approaches. According to Nielsen et al. [4], any of the current inspection and maintenance practices within the Australian rail bridge industry doesn't have capability to predict the future conditions of bridge components and Australia's rail bridges seem lack of historical inspection data. Decision making procedure is subjective and it doesn't optimize the cost. Dealing with thousands of bridges has become a real challenge for bridge engineers and managing of these structures is extremely difficult without having a systematic way for decision making. Currently projects are undergoing to implement a bridge maintenance system (BMS) for rail bridges in Australia and thus best suitable deterioration models which match with current inspection and maintenance regimes are vital parts for solving the above discussed issues. This is the motivation of this study.

Markov chain approach is the most popular network level stochastic deterioration modeling technique that has been intensively used for predicting the future conditions of network level infrastructure facilities [3]. It uses available current condition rating data for predicting the future condition states while capturing the physical, model and statistical uncertainties [4]. Since Australia's rail bridge network lacks historical data, Markov approach is suitable for developing network level deterioration models compared to neural network and risk based models. Markov models can be subdivided into state-based models and time-based models. 
Due to high variability of field data collected and current maintenance records of the condition state of bridge components over constant inspection period, use of discrete time sate-based deterioration models are more realistic than time-based one [5]. Therefore, discrete state Markov models are selected to establish deterioration models in this study. Main task of the Markov model here is to estimate the transition probability matrix with limited inspection data, which is also known as calibration of Markov models [6]. If Transition Probability (TP) and initial condition are known for a given component group, the future condition states can be easily obtained by Markov chain method.

Most widely used Markov-model calibration technique is regression based nonlinear optimization approach [5]. In this method, the bridge performance curve is first obtained using linear or nonlinear regression analysis for assumed function type, normally a $3^{\text {rd }}$ order polynomial [ $\left.2 \& 5\right]$. Secondly, constrained nonlinear optimization method is applied to minimize the sum of absolute distances between regression performance function values and related expected performance function values obtained by Markov formula which is also known as minimizing the objective function. Finally, main elements in transition probability matrix are supposed to be obtained at global minimum point of nonlinear objective function. Methodology related to distribution based nonlinear optimization is also quite similar to regression based nonlinear optimization and the only difference is objective function that is used for the analysis, as the sum of absolute differences between distribution of condition obtained from the field data and the distribution given in the condition state vector from Markov equation. However, both of the nonlinear optimization methods discussed above have some common drawbacks: 1) It may stop at local minimum points resulting in incorrect transition probability values; 2) cannot provide confidence limits of the transition probabilities and 3) difficult to update when new data are available [6]. Furthermore, accuracy of the regression based nonlinear optimization method is solely dependent on assumed function type that is selected for the regression fitting. Micevski [3] and Tran [6] have successfully used Markov chain Monte Carlo (MCMC) method for pipe deterioration modeling by overcoming the above mentioned limitations. Therefore, in this study, MCMC is applied to railway bridge deterioration modeling.

\subsection{Markov Approach to Bridge Deterioration Modeling}

\subsubsection{Factor Identification}

Bridge components deteriorate with time and deterioration rates and patterns may vary with contributing factors such as age, rail-traffic volume (Tonnage passes on bridge for given time), span, number of tracks, material type, functional classification (passenger train bridges or freight train bridges), nature of the defect, struc- 
ture type and environmental categories, etc. These factors were identified based on most common contributing factors that were considered in previous studies [1], [5] $\&$ [2] and through considering expert opinions of rail bridge engineers.

\subsubsection{Markov Approach}

The Markov chain is a special case of the Markov process and generally a discrete-time stochastic process $\left\{X_{(t)}, t=0,1,2, \ldots\right\}$ that takes on a finite or countable number of possible discrete states. This can be modeled as a serious of transitions between certain states. For an example, according to condition rating system of a large railway company in Australia, condition state of a bridge component can be defined by an integer between 1 and 5 , where 1 represents the structure is in its best condition possible and 5 represents the maximum condition state before a bridge/component is repaired or replaced. It is assumed that whenever the process is in certain state $i$, there is a fixed conditional probability $p_{i j}$ that a component will be in state $j$ in one time unit later and can be expressed as discrete parameter stochastic process as given in equation (1.1). In homogenous Markov process, it is assumed that the conditional probability does not change over given time. Therefore, equation (1.1) can be deduced to equation (1.2) with all 5 states of $i$ and $j$ for all $t$ 's.

$$
\begin{aligned}
p_{i j} & =\mathrm{P}\left\{X_{t+1}=j \mid X_{(t)}=i, X_{(t-1)}=i_{t-1}, \ldots . ., X_{1}=i_{1}, X_{(0)}=i_{0}\right\}, \\
p_{i j} & =\mathrm{P}\left\{X_{t+1}=j \mid X_{(t)}=i\right\} .
\end{aligned}
$$

These probabilities are represented in matrix form that is called Transition Probability Matrix (TPM or P) of the Markov chain. For example, according to five possible condition states, it yields a $5 \times 5$ matrix as given bellow.

$$
\mathrm{P}=\left[\begin{array}{lllll}
p_{11} & p_{12} & p_{13} & p_{14} & p_{15} \\
p_{21} & p_{22} & p_{23} & p_{24} & p_{25} \\
p_{31} & p_{32} & p_{33} & p_{34} & p_{35} \\
p_{41} & p_{42} & p_{43} & p_{44} & p_{45} \\
p_{51} & p_{52} & p_{53} & p_{54} & p_{55}
\end{array}\right]
$$

Each element in the TPM represents the probability of transition from one state to another for one inspection period. Sum of the each raw of the TPM is equal to one from total probability theorem. Without rehabilitation or repair work, bridge components would be gradually deteriorating and thus, the bridge component condition ratings either upgrade to a higher number or remain unchanged in one 
inspection period [2]. Hence, the probability $p_{i j}$ is null for $i>j$ where $i$ and $j$ are condition states of the Markov model. Furthermore, in many studies [2] \& [5], it was assumed that bridge component condition rating would not be upgraded by more than one state within one single year, or probability of upgrade to more than one state within one year is assumed to be zero. Therefore, one year TPM can be simplified further.

However, in two-year TPM, multi state transition events are automatically generated according to Markov property. This two-year TPM is equal to the second power of one year TPM [5].

Percentage of bridges/components in each condition rating after $t$ years of age for a selected group can be expressed as a raw vector which is defined as the condition state vector $\left(\mathrm{C}_{(t)}\right)$ and expressed as $\mathrm{C}_{(t)}=\left[\mathrm{C}_{1(t)} \mathrm{C}_{2(t)} \mathrm{C}_{3(t)} \mathrm{C}_{4(t)} \mathrm{C}_{5(t)}\right]$ where $\mathrm{C}_{\mathrm{i}(t)}$ is percentage of bridge components in condition rating $\mathrm{i}$ (for $\mathrm{i}=1,2,3,4,5$ ) after $t$ years. Furthermore, condition state matrix after 0 years (when $t=0$ ) is known as the initial condition state vector which is notated as $\mathrm{C}_{(0)}$. If initial time is chosen as age $=0$, just after construction of component/(s), it's obvious all components are in condition rating 1 (best condition). Thus, $\mathrm{C}_{(0)}=\left[\begin{array}{lllll}1 & 0 & 0 & 0 & 0\end{array}\right]$. If transition probability matrix (TPM) and initial condition state matrix $\left(\mathrm{C}_{(0)}\right)$ are known, condition state matrix after time $t$ can be obtained by the multiplication of initial condition state matrix by $t^{\text {th }}$ power of TPM by using Chapman-Kolmogorov formula as follows.

$$
\mathbf{C}_{(t)}=\mathbf{C}_{(0)} \times \mathbf{P}^{t}
$$

Since $\mathrm{C}_{(0)}$ is frequently known parameter for determining the future condition states, the real challenge is to estimate Transition Probability Matrix (TPM) for a given component group. Different statistical methods have been applied in past studies to estimating TPM of infrastructure facilities including bridges, pipe lines, pavement systems, etc. Estimating of TPMs is also known as calibrating the Markov chains [6] and discussed in next section.

Performance index is defined by $\mathrm{PI}=6-\mathrm{CR}$ where $\mathrm{CR}$ is the condition rating. Without a repair or replacement of a given component, PI decreases as the component age increases.

If the condition state matrix after time $t$ is known, expected value of facility condition at time $t$ can be calculated and defined as Expected Performance Index (EPI) [6].

$$
\mathrm{EPI}_{(t)}=\mathbf{C}_{(t)} \times \mathbf{S}
$$

where, $\mathbf{C}_{(t)}$ is condition state vector given in equation (1.4) and $\mathbf{S}$ is a column vector with condition ratings that is the transpose of matrix of [ [ $\left.\begin{array}{lllll}5 & 4 & 3 & 2 & 1\end{array}\right]$. 


\subsection{Calibrating the Markov Model by MCMC}

\subsubsection{Bayesian Approach}

Let us consider a set of data (condition ratings) for a bridge element group as $\mathrm{Y}$ $=\left\{\mathrm{y}_{1}, \mathrm{y}_{2}, \mathrm{y}_{3}, \ldots . ., \mathrm{y}_{\mathrm{n}}\right\}$ and $\theta$ represent unknown model parameter vector (in here unknown elements $p_{i j}$ in TPM). The joint probability distribution $\mathrm{P}(\mathrm{Y} / \theta)$ is known as the sampling distribution or likelihood function which should be a known parameter to perform any inference. $\mathrm{P}(\theta / \mathrm{Y})$ is known as the posterior distribution or target distribution and $\mathrm{P}(\theta)$ is called prior distribution of unknown model parameter. According to Bayes' rule for known value of data Y, posterior distribution of model parameter is given by:

$$
\mathrm{P}(\theta / \mathrm{Y}) \propto \mathrm{P}(\theta) \mathrm{P}(\mathrm{Y} / \theta)
$$

When it applies to Markov calibrating model, likelihood function of unknown transition probability density vector for a given bridge data set $Y=\left\{y_{1}, y_{2}, y_{3}\right.$, $\left.\ldots . . ., \mathrm{y}_{\mathrm{n}}\right\}$ can be easily derived by using joint probability theory and deduced into logarithmic form for easy computation [6] as:

$$
\log [\mathrm{L}(\mathrm{Y} / \theta)]=\sum_{t=1}^{T} \sum_{i=1}^{5} N_{i}^{t} \log \left(C_{i t}\right)
$$

where $L(Y / \theta)$ is the likelihood to observe a condition rating data set $Y=\left\{y_{1}, y_{2}\right.$, $\left.\mathrm{y}_{3}, \ldots . ., \mathrm{y}_{\mathrm{n}}\right\}$ for given bridge element group with $n$ total records, $t$ is the bridge element age in years, $T$ is the largest age found in the data set and $N_{i}^{t}$ is the number of elements in condition $i$ at year $t ; C_{i t}$ is the probability in condition state $i$ at year $t$ and can be expressed as a function of TPM by using equation (1.4).

\subsubsection{MCMC simulation method with MHA}

Markov Chain Monte Carlo (MCMC) methods have been increasingly used in recent years for simulating complex, nonstandard multivariate distributions [3].The Metropolis-Hasting Algorithm (MHA) is the most popular example of a MCMC method and recently used for many engineering applications [6]. According to equation (1.6), posterior density of transition probability values is proportional to multiple of prior density into likelihood function. This property is used in this analysis to allow MCMC method with Metropolis Hasting Algorithm (MHA) to generate samples from posterior distribution [6]. When applying MHA, it's required to choose a proposal density $\mathrm{q}(x, y)$ where $\int q(x, y) d y=1$, for sampling 
from the target distribution [6]. Although, the proposal distribution is arbitrarily chosen from some family distributions, performances are dependent on how much the selected distribution approximates the posterior. "The candidate-generating density depends on the current state of the Markov chain, which means that when a process is at the point $x$ this density generates a point $y$ from $\mathrm{q}(x, y)$. The new point y is always accepted, otherwise, $y$ can be accepted with a probability $\alpha(x, y)$. In other words, if the jump goes 'uphill', it is always accepted; if 'downhill', it is accepted with a non-zero probability" [6].

\subsection{Case Study}

According to data availability and considering contribution factors such as material, Average tonnage passes per week, Environmental categories, etc., condition rating data of 40 transverse timber bridge decks in major inland railway lines, over past 15 years were selected to do this analysis.

A major problem identified from data analysis is related to subjective nature of bridge inspections. Veshosky [7] argued that condition ratings that are assigned by different inspectors for same bridge component potentially could result in different values. This problem has been addressed up to some extent by conducting workshops and training programs for inspectors, reviewing and adjusting the condition rating data by supervisor and by conducting detailed inspections by experienced engineers. Usually, all bridges in a one railway line are inspected by same inspector. Thus, consideration of condition rating data of bridges in one railway line for this analysis also helps to control subjective nature of the bridge inspection records based on assumption that inspection procedures and rating criteria are approximately same than across many railway lines. However, each railway line does not have significant number of bridges and hence this approach is unable to apply for each line. In this case, analysis has to be done by combining bridges in different railway lines with similar characteristics based on assumption that the observed bridge condition rating are randomly distributed about their true values.

In this study, deterioration models were developed for railway bridge decks with no improvement works have been undertaken in between. Therefore, inspection records for bridge deck element, after repair and reconstruction actions, have been removed from analysis data base. However, it has been identified that every repair and maintenance work has not been recorded in bridge inventory. Some Bridge deck ratings figured improvement of condition with time. However, unless repair or maintenance work is done, bridge components would be gradually deteriorating so that the bridge condition rating is either unchanged or upgraded into a higher number according to the condition rating system. Relaying on that assumption, bridge deck element whose condition rating had been improved over the years were identified and also removed from data base. Furthermore, very good condition rating values have been observed for relatively old bridge decks ele- 
ments. This could be happen due to unrecorded repair or reconstruction work before 15 years back, since bridge agency has only 15 years back inspection records. Moreover, some of the newly constructed bridge components had not been assigned in to condition rating in one category. Madanat [1] argued that this could be due to inadequate initial design or construction or misapplication of rating procedure by bridge inspectors. It was also identified that double counting of same records also exists. Hence, these unusual condition rating data were filtered and rejected from the analysis data base. Finally, 242 total records were obtained after filtration process. For statistical validation and comparison purpose, data set was split randomly such that $75 \%$ as calibrated data set and the rest as the test data set. Calibrated data set was used for analysis first and validated with test data set by using Chi-square test [6].

\subsubsection{Analysis Results}

MATLAB programming codes were developed with MHA algorithm for MCMC. The MHA was run with 50,000 iterations for the calibration data set and later for entire data set until the transition probability values converge to stationary distributions after first 15000 warm-up runs. Variance covariance matrix was adjusted until acceptance rate becomes near to optimum acceptance rate of 0.234 . Fig. 1.1 shows the trace plots after 20000 iterations with no warm-up runs for P11, P22, P33 \& P44. It's clear that after 2000 iterations, all transition probability values are approximately convergent to stationary distributions. Trace plots with 50000 iterations after 15000 burnings (Warm-up runs) are given in Fig. 1.2. Standard deviation for each transition probability values were found very small and given in Table 1.2 in Section 1.4.2. Mean values of each TPM elements are convergent to constants as shown in Fig. 1.3. Finally, mean values are obtained for the transition probability matrixes for one year transition period and two years transition period, respectively, see, equations (1.8) and (1.9). By using estimated TPM and known initial condition state vector, Markov equation is applied to obtaining the future condition state proportions with time elapsing as given in Fig. 4.4. 

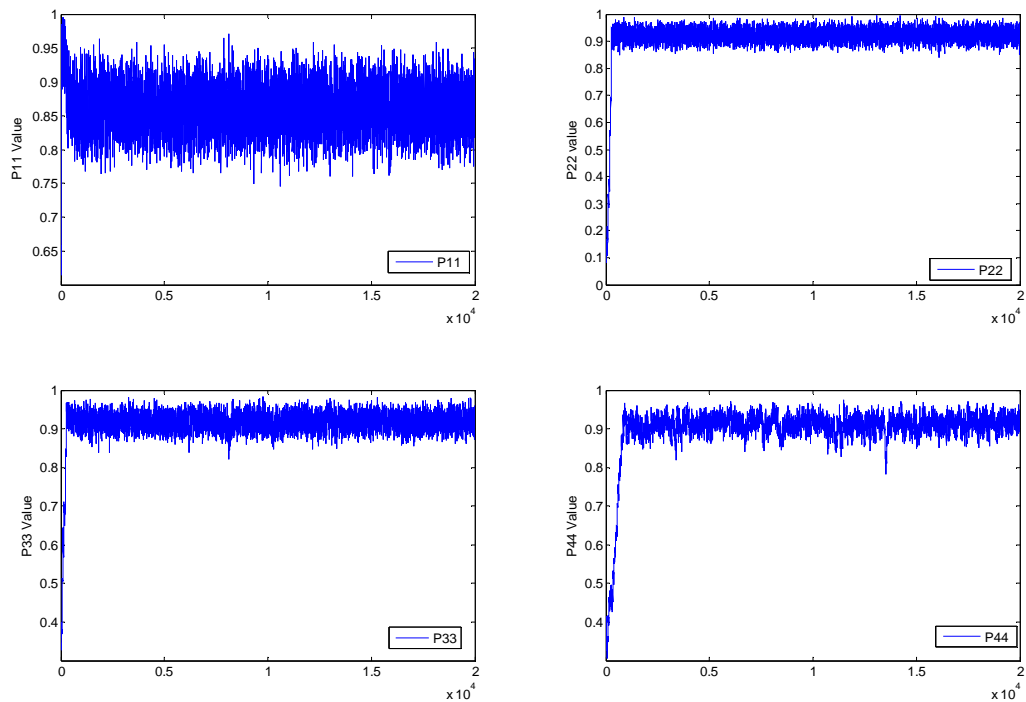

Fig. 1.1 Trace plots for main transition probability values after first 20000 iterations
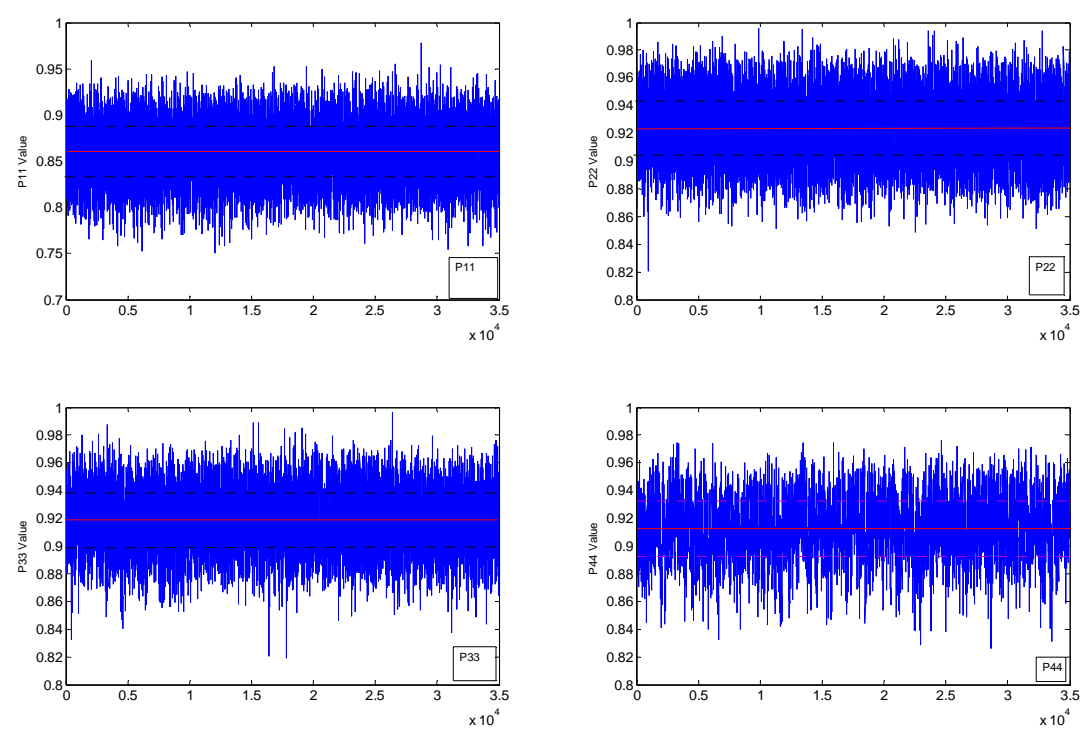

Fig. 1.2 Trace plots for 50,000 iterations with 15,000 warm-up runs for main TPM values 

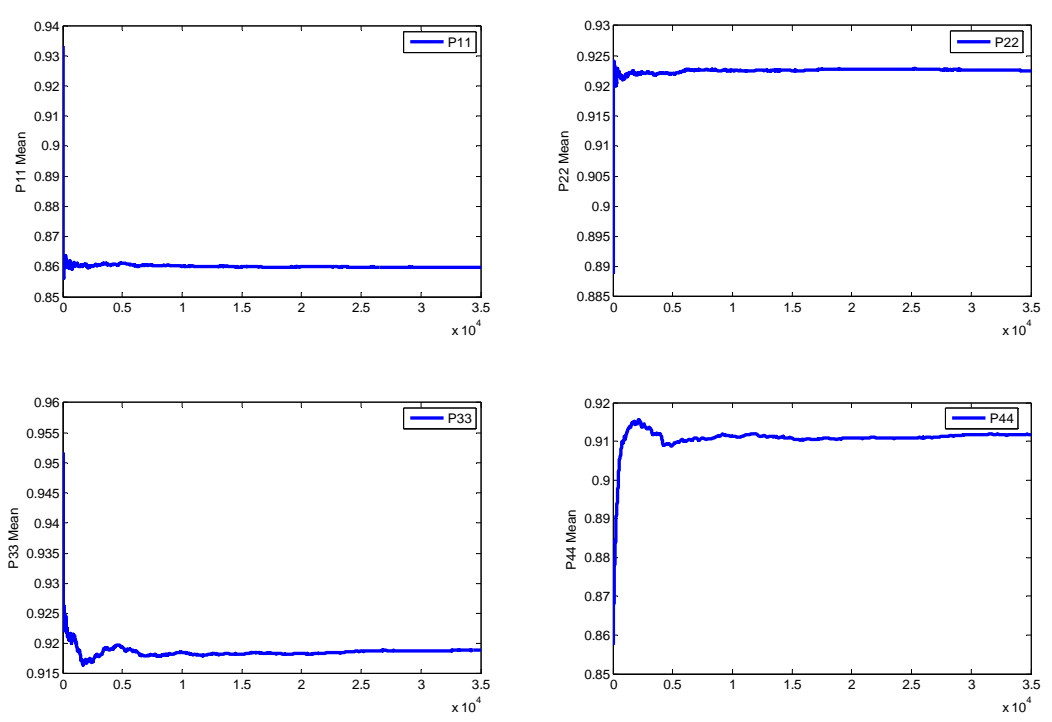

Fig. 1.3 Trace plots for mean transition probability values after for 50,000 iterations with 15,000 warm-up runs

$\mathrm{P}=\left[\begin{array}{ccccc}0.86 & 0.14 & 0 & 0 & 0 \\ 0 & 0.92 & 0.08 & 0 & 0 \\ 0 & 0 & 0.92 & 0.08 & 0 \\ 0 & 0 & 0 & 0.91 & 0.09 \\ 0 & 0 & 0 & 0 & 1\end{array}\right]$

$\mathrm{P}=\left[\begin{array}{ccccc}0.7496 & 0.2492 & 0.0112 & 0 & 0 \\ 0 & 0.8464 & 0.1472 & 0.0064 & 0 \\ 0 & 0 & 0.8464 & 0.1464 & 0.0072 \\ 0 & 0 & 0 & 0.84281 & 0.1719 \\ 0 & 0 & 0 & 0 & 1\end{array}\right]$




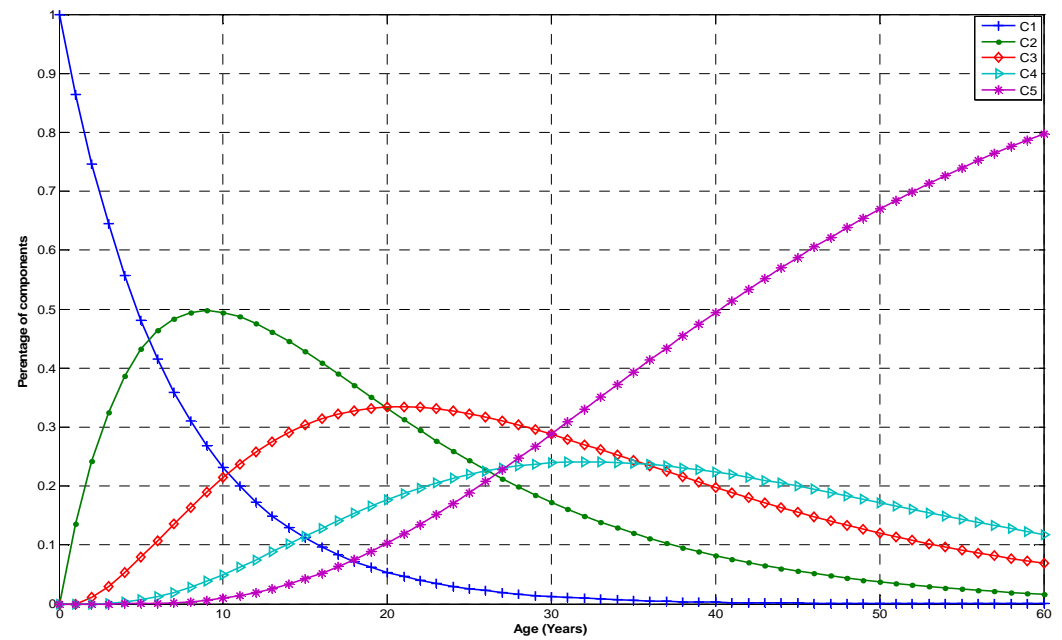

Fig. 1.4 Condition percentage distribution of each condition sate vs. age in MCMC with MHA for entire data set

\subsubsection{Verification of Results}

Model was validated by using Goodness-of-fit test using Chi-squared test statistics $\left(\chi^{2}\right)$ which is based on null hypothesis that the observed number of bridge elements is matched with the predicted number of elements in different condition states [6]. 95\% confidence level was used to evaluate the fitness of the models. Chi-squared test statistics $\left(\chi^{2}\right)$ for bridge element deterioration models in this study was calculated according to equation (1.10).

$$
\chi^{2}=\sum_{i=1}^{5} \frac{\left(O_{i}-P_{i}\right)^{2}}{P_{i}}
$$

where, $O_{i}$ is observed number of transoms in condition $i$ (transom is the main element of transverse timber bridge deck), $P_{i}$ is predicted number of transoms in condition $i$.

Table 1.1 Chi-square values of MCMC method results

\begin{tabular}{c|cc}
\hline \multirow{2}{*}{ Method } & \multicolumn{2}{|c}{ Chi-Square values with 4 degree of freedoms $(\leq 9.49)$} \\
\cline { 2 - 3 } & Calibrated data set & Test data set \\
\hline MCMC simulation with MHA & 0.268 & 1.508 \\
\hline
\end{tabular}


Table 1.2 Main transition probability values and $95 \%$ confidence intervals for entire dataset

\begin{tabular}{ccccc}
\hline$p$ value & Mean & STD & Upper limit & Lower limit \\
\hline P11 & 0.864 & 0.02564 & 0.867 & 0.860 \\
P22 & 0.921 & 0.01651 & 0.923 & 0.918 \\
P33 & 0.923 & 0.0178 & 0.925 & 0.920 \\
P44 & 0.913 & 0.0195 & 0.915 & 0.910 \\
\hline
\end{tabular}

\subsection{Conclusions}

This paper reviews the application of Markov Chain Monte Carlo (MCMC) approach with Metropolis Hasting Algorithm (MHA) for network level bridge deterioration modeling. From expert opinions and previous studies, contribution factors for rail bridge deterioration were identified. Bridge inventory data were collected from a main industrial partner in Australia and reviewed. Transition Probability Matrix (TPM) was estimated by using MCMC with MHA for bridge deck transoms with similar characteristics. The outcome of the MHA is sample distributions for transition probabilities which increases the chance of capturing true global optimum compared to Regression based NOA methods. Outputs of the deterioration models were validated by using goodness-of-fit test. Results show that Chi-square values of transition probabilities for calibrated and test data set are well below the limit values. Obtaining very small Chi-square values compared to limit value convinced the superiority of the MHA and MCMC for bridge deterioration modeling. Ability to express confidence intervals for transition probabilities is another advantage of MCMC method, over conventional Markov calibration methods such as regression based and distribution based NOA. Major drawback of proposed methodology over NOA is that MCMC seeks considerable number of condition rating data which expands the age range of the selected component group.

Further Work: Available Markov calibration techniques and MCMC will be applied to developing a network level deterioration models for other bridge components in order to make a further comparison with other methods.

\section{Acknowledgement}

This research is funded by CRC for Rail Innovation Australia on Railway Asset Management Research Program. 


\subsection{References}

1. Madanat S, Mishalani R, Wan Ibrahim W H (1995) Estimation of infrastructure transition probabilities from condition rating data. J. Infrastruct. Sys., ASCE 1(2):120-125

2. Jiang Y, Saito M, Sinha K C (1988) Bridge performance prediction model using the Markov chain. Transportation Research Record 1180, Transportation Research Board, Washington, D.C., $25-32$

3. Micevski T, Kuczera G, Coombes P (2002) Markov Model for Storm Water Pipe Deterioration. Journal of Infrastructure Systems, ASCE 8(2):49-56

4. Nielsen D, Chattopadhyay G, Dhamodharan, R (2012) Life cycle management of railway bridges: defect management. Conference on Railway Engineering, 10-12 September 2012, Brisbane, Australia, p.425-434

5. Morcous G (2006) Performance prediction of bridge deck systems using Markov chains. Journal of Performance of Constructed Facilities 20:146-155

6. Tran H D (2007) Investigation of deterioration models for stormwater pipe systems. School of Architectural, Civil and Mechanical Engineering. Victoria University, Australia

7. Veshosky D, Beidleman C R (1996) Closure to "Comparative Analysis of Bridge Superstructure Deterioration”. J. Struct. Eng. 122(6):710-71 